\title{
Delay estimation via sliding mode for nonlinear time-delay systems
}

\author{
Gang Zheng ${ }^{\mathrm{a}, \mathrm{b}}$, Andrey Polyakov ${ }^{\mathrm{a}, \mathrm{b}}$, Arie Levant ${ }^{\mathrm{c}}$ \\ ${ }^{a}$ INRIA - Lille Nord Europe, 40 avenue Halley, Villeneuve d'Ascq 59650, France. \\ ${ }^{b}$ CRIStAL, CNRS UMR 9189, Ecole Centrale de Lille, BP 48, 59651 Villeneuve dAscq, France. \\ ${ }^{c}$ Tel-Aviv University, Israel
}

\begin{abstract}
This paper deals with delay identifiability and delay estimation for a class of nonlinear time-delay systems. The theory of noncommutative rings is used to analyze the identifiability. In order to estimate the delay, a sliding mode technique and a classical Newton method are combined to show the possibility to have a local (or global) delay estimation for periodic (or aperiodic) delay signals.
\end{abstract}

Keywords: Time-delay; Identification; Sliding mode

\section{Introduction}

Time-delay systems are widely used to model concrete systems in engineering sciences, such as biology, chemistry, mechanics and so on Kolmanovskii and Myshkis (1999); Niculescu (2001). Many results have been reported for the purpose of stability and observability analysis, by assuming that the delay of the studied systems is known. It makes the delay identification one of the most important topics in the field of time-delay systems.

Delay identifiability has been widely studied in the literature for linear time-delay system. However, for a nonlinear time-delay system, this issue is not trivial, and we will borrow the concept of non-commutative rings to analyze it. The theory of non-commutative rings was firstly proposed by Moog et al. (2000) for the disturbance decoupling problem of nonlinear time-delay systems. Then this method was applied to study observability of nonlinear time-delay systems with known inputs in Xia et al. (2002), to analyze parameter identifiability for nonlinear time-delay systems in Zhang et al. (2006), and to study state elimination and delay identification of nonlinear time-delay systems in Anguelova and Wennberg (2008); Zheng et al. $(2011,2013)$.

Concerning the techniques to identify the delay, up to now, various methods have been proposed, and a nice survey can be found in Bjorklund and Ljung (2003). Briefly, Tuch et al. (1994); Diop et al. (2001) and Ren et al. (2005) used the least squares method or its variation to estimate the delay for linear time-delay systems. An adaptive identification method was proposed in Orlov et al. (2003) for identification of both parameters and delay. By using a convolution approach, Belkoura (2005) and Belkoura et al. (2009) proposed an algebraic method

\footnotetext{
This paper was supported in part by Ministry of Higher Education and Research Nord-Pas de Calais Regional Council, by FEDER through the Contrat de Projets Etat Region (CPER), France.

Email address: gang . zheng@inria.fr (Gang Zheng)
}

to identify the delay involved in a linear time-delay system. In Drakunov et al. (2006), a variable structure observer was proposed to estimate the delay. More recently, a recursive gradient method was proposed in Barbot et al. (2012) to estimate the delay for a nonlinear time-delay system.

Comparing with the existing identification methods, the main contributions of this paper are twofold. Firstly, this paper investigates the delay identification problem for a class of nonlinear time-delay systems while most of existing works in the literature are for a linear time-delay system. Secondly, compared with the existing results which assume the delay should be involved in aperiodic trajectory, our method combines the high order sliding mode technique Levant (2003) and classical Newton method Saupe (1988) to identify the delay, and the proposed method can also treat periodic case.

This paper is an extension of Zheng et al. (2016), and is organized as follows. Section 2 states the systems and the problem which will be studied in this paper. The delay identifiability property will be analyzed in Section 3. This property is based on the algebraic framework proposed in Xia et al. (2002). By using a high order sliding mode technique, an estimator is proposed in Section 4 to identify the delay. This identification is local if the delay signal is periodic. Finally the proposed result is applied to analyze the identifiability for an illustrative example in Section 5.

\section{Problem statement}

It is assumed that the delays are constant and commensurate, that is all of them are multiples of an elementary unknown delay $\tau$. Under this assumption, the considered nonlinear time-delay system is described as follows:

$$
\left\{\begin{array}{l}
\dot{x}=f(x(t), x(t-\tau), \cdots, x(t-s \tau)) \\
y=h(x(t), x(t-\tau), \cdots, x(t-s \tau)) \\
x(t)=\psi(t), t \in[-s \tau, 0]
\end{array}\right.
$$


$x \in \mathbb{R}^{n}, y \in \mathbb{R}$ is the measurable output; $f$ and $h$ are meromorphic functions ${ }^{1}$ which are functions of variables $\{x, x(t-\tau), \cdots, x(t-s \tau)\} ; \psi:[-s \tau, 0] \rightarrow \mathbb{R}^{n}$ denotes unknown continuous initial functions.

Assumption 1. For the initial function $\psi$, the system (1) admits a unique smooth solution, which is bounded on the compact set $W$, i.e. $x \in W \subset \mathbb{R}^{n}$.

Unlike conventional control systems, the studied system (1) does not contain an input (control) $u$. The choice of such a form (1) is motivated by the fact that the input signal is normally known and it is function of $x$. Therefore, the closed-loop system might be written as (1) for which this paper focuses on the identification of the unknown but constant elementary delay $\tau$.

\section{Identifiability analysis}

For a nonlinear time-delay system described in (1), the analysis of delay identifiability is not trivial as that for the linear case, where commutative algebra can be applied. For nonlinear case, we have to use the non-commutative algebraic framework introduced in Xia et al. (2002), which will be firstly recalled in the following section.

\subsection{Algebraic framework}

Denote by $\mathscr{K}$ the field of meromorphic functions of a finite number of the variables from $\left\{x_{j}(t-i \tau), j \in[1, n], i \in[0, s]\right\}$. For the sake of simplicity, introduce the delay operator $\delta$, which means, for $i \in \mathbb{Z}^{+}$:

$$
\begin{gathered}
\delta^{i} \xi(t)=\xi(t-i \tau), \xi(t) \in \mathscr{K}, \\
\delta^{i}(a(t) \xi(t))=\delta^{i} a(t) \delta^{i} \xi(t) \\
=a(t-i \tau) \xi(t-i \tau) .
\end{gathered}
$$

Let $\mathscr{K}(\delta]$ denote the set of polynomials in $\delta$ over $\mathscr{K}$ of the form

$$
a(\delta]=a_{0}(t)+a_{1}(t) \delta+\cdots+a_{r_{a}}(t) \delta^{r_{a}}
$$

where $a_{i}(t) \in \mathscr{K}$ and $r_{a} \in \mathbb{Z}^{+}$. The addition in $\mathscr{K}(\delta]$ is defined as usual, but the multiplication is given as:

$$
a(\delta] b(\delta]=\sum_{k=0}^{r_{a}+r_{b}} \sum_{i+j=k}^{i \leq r_{a}, j \leq r_{b}} a_{i}(t) b_{j}(t-i \tau) \delta^{k}
$$

Thanks to the definition of $\mathscr{K}(\delta],(1)$ can be rewritten in a more compact form:

$$
\left\{\begin{array}{l}
\dot{x}=f(x, \delta) \\
y=h(x, \delta) \\
x(t)=\psi(t), t \in[-s \tau, 0]
\end{array}\right.
$$

where $f(x, \delta)=f(x(t), x(t-\tau), \cdots, x(t-s \tau))$ and $h(x, \delta)=$ $h(x(t), x(t-\tau), \cdots, x(t-s \tau))$, with entries belonging to $\mathscr{K}$.

\footnotetext{
${ }^{1}$ means quotients of convergent power series with real coefficients Conte et al. (1999); Xia et al. (2002).
}

With the standard differential operator $d$, denote by $\mathscr{M}$ the left module over $\mathscr{K}(\delta]$ :

$$
\mathscr{M}=\operatorname{span}_{\mathscr{K}(\delta]}\{d \xi, \xi \in \mathscr{K}\}
$$

where $\mathscr{K}(\delta]$ acts on $d \xi$ according to (2) and (3). Note that $\mathscr{K}(\delta]$ is a non-commutative ring, however it is proved that it is a left Ore ring Ježek (1996); Xia et al. (2002), which enables to define the rank of a left module over $\mathscr{K}(\delta]$.

\subsection{Output delay equation}

In order to study the delay identifiability of (6), we need to first deduce a certain output delay dependent equation, based on which the identifiability can be then analyzed.

Definition 1. Zheng and Richard (2016) For system (6), an equation with delays, containing only the output and a finite number of its derivatives:

$$
\alpha\left(h, \dot{h} \ldots, h^{(k)}, \delta\right)=0, k \in \mathbb{Z}^{+}
$$

is said to be an output delay equation (of order $k$ ). Moreover, this equation is said to involve the delay in an essential way for (6) if it cannot be written as $\alpha\left(h, \dot{h} \ldots, h^{(k)}, \delta\right)=$ $a(\delta] \tilde{\alpha}\left(h, \dot{h} \ldots, h^{(k)}\right)$ with $a(\delta] \in \mathscr{K}(\boldsymbol{\delta}]$.

If there exists an output equation involving the delay in an essential way, then the delay might be identifiable if certain sufficient conditions are satisfied. Thus, the first task is to seek such an output delay equation. In what follows, we define the derivative and Lie derivative for nonlinear time-delay systems from the non-commutative point of view.

For $0 \leq j \leq s$, let $f(x(t-j \tau))$ with $f_{r} \in \mathscr{K}$ for $1 \leq r \leq n$ and $h(x(t-j \tau)) \in \mathscr{K}$, define

$$
\frac{\partial h}{\partial x_{r}}=\sum_{j=0}^{s} \frac{\partial h}{\partial x_{r}(t-j \tau)} \delta^{j} \in \mathscr{K}(\delta] .
$$

then the Lie derivative for nonlinear systems without delays can be extended to nonlinear time-delay systems in the framework of Xia et al. (2002) as follows

$$
\frac{d h}{d t}=L_{f} h=\frac{\partial h}{\partial x}(f)=\sum_{r=1}^{n} \sum_{j=0}^{s} \frac{\partial h}{\partial x_{r}(t-j \tau)} \delta^{j}\left(f_{r}\right)
$$

Based on the above notations, one can define the observability indices introduced in Krener (1985) over non-commutative rings. For $1 \leq k \leq n$, let $\mathscr{F}_{k}$ be the following left module over $\mathscr{K}(\delta]:$

$$
\mathscr{F}_{k}:=\operatorname{span}_{\mathscr{K}(\delta]}\left\{d h, d L_{f} h, \cdots, d L_{f}^{k-1} h\right\}
$$

It was shown in Zhang et al. (2006) that the filtration of $\mathscr{K}(\delta]$ module satisfies $\mathscr{F}_{1} \subset \mathscr{F}_{2} \subset \cdots \subset \mathscr{F}_{\rho}$, and it is stationary for a certain $\rho \leq n$, thus $\rho$ is called the observability index of the output $y$.

Define

$$
£=\operatorname{span}_{\mathbb{R}[\delta]}\left\{h, \cdots, L_{f}^{\rho-1} h\right\}
$$


where $\mathbb{R}[\boldsymbol{\delta}]$ is the commutative ring of polynomials in $\delta$ with coefficients belonging to the field $\mathbb{R}$. Note

$$
\Phi_{h}(x, \delta)=\left(h, L_{f} h, \cdots, L_{f}^{\rho-1} h\right)^{T}
$$

since $\rho \leq n$, which implies that $d \Phi_{h}(x, \delta)$ is linearly independent vectors over $\mathscr{K}(\delta]$.

Due to the fact that the observability index $\rho \leq n$, there exist $\omega_{i} \in £(\delta]$ for $1 \leq i \leq \rho$ such that

$$
L_{f}^{\rho} h=\sum_{i=1}^{\rho} \omega_{i} L_{f}^{i-1} h, \omega_{i} \in £(\delta]
$$

which is exactly an output delay equation.

Assumption 2. It is assumed that system (6) has the observability index $\rho \leq n$ such that

$$
\operatorname{rank}_{\mathscr{K}(\delta]} \frac{\partial\left\{h, L_{f} h, \cdots, L_{f}^{\rho-1} h\right\}}{\partial x}<\operatorname{rank}_{\mathscr{K}} \frac{\partial\left\{h, L_{f} h, \cdots, L_{f}^{\rho} h\right\}}{\partial x}
$$

Theorem 1. Anguelova and Wennberg (2008) Under Assumption 1, the output equation (10) involves the delay in an essential way if and only if (11) is satisfied.

Remark 1. It has been shown in Anguelova and Wennberg (2008) that if (11) is not satisfied, then an original system is equivalent to a delay-free system via a change of coordinates. In other words, for a concrete system containing the delay, (11) can be used to check whether the studied system is a 'real' timedelay system or not.

For $\Phi_{h}(x, \delta) \in \mathscr{K}^{\rho}$, since $\operatorname{rank}_{\mathscr{K}(\delta]} d \Phi_{h}(x, \delta)=\rho$, thus it is linearly independent over $\mathscr{K}(\delta]$. Therefore there exist $(n-\rho)$ functions $\Phi_{\xi}(x, \delta)$ such that

$$
\left[\begin{array}{l}
z \\
\xi
\end{array}\right]=\Phi(x, \delta)=\left[\begin{array}{c}
\Phi_{h}(x, \delta) \\
\Phi_{\xi}(x, \delta)
\end{array}\right]
$$

forms a change of coordinates, satisfying $\operatorname{rank}_{\mathscr{K}(\delta]} \frac{\partial \Phi(x, \delta)}{\partial x}=n$. It is obvious that $\operatorname{dim} \xi=0$ for the case $\rho=n$. With this change of coordinates, system (6) can be written into the following canonical form:

$$
\left\{\begin{array}{l}
\dot{z}_{1}=z_{2} \\
\vdots \\
\dot{z}_{\rho}=\sum_{i=1}^{\rho} \omega_{i} z_{i} \\
\dot{\xi}=\beta(z, \xi, \delta) \\
y=C z=z_{1}
\end{array}\right.
$$

where $z=\left(z_{1}, \cdots, z_{\rho}\right)^{T}=\left(h, \cdots, L_{f}^{\rho-1} h\right)^{T}, \omega_{i} \in £(\delta], C=$ $(1,0, \cdots, 0)$ and $\beta \in \mathscr{K}^{(n-\rho) \times 1}$ represents the unobservable dynamics.

\subsection{Identifiability}

Consider the $\rho$ th equation of (13), since $\omega_{i} \in £(\delta]$ where $£(\delta]$ is the set of polynomials in $\delta$ with coefficients over $£$ defined in (9), therefore the maximal degree of $\delta$ in $\omega_{i}$ is bounded as

$$
\operatorname{deg}_{\delta} \omega_{i} \leq i \times s
$$

where $s$ is the maximal number of delays involved in system (1). For the sake of simplicity, note

$$
d_{\max }=\max \left\{\operatorname{deg}_{\delta} \omega_{i}, \text { for } 1 \leq i \leq \rho\right\} \leq \rho \times s
$$

the $\rho$ th equation of (13) can be rewritten as

$$
\dot{z}_{\rho}(t)=\sum_{i=0}^{d_{\max }} p_{i}\left(z_{1}(t-i \tau), \cdots, z_{\rho}(t-i \tau)\right)=P(z, t, \tau)
$$

where $p_{i}\left(z_{1}(t-i \tau), \cdots, z_{\rho}(t-i \tau)\right)$ represents all terms containing $(t-i \tau)$. Let us now introduce the following distinguishability definition for different delays.

Definition 2. For system (13), two different delays $\tau>0$ and $\bar{\tau}>0$ is said to be distinguishable if there exists an interval $T>0$ such that

$$
\int_{t-T}^{t}[P(z, \sigma, \tau)-P(z, \sigma, \bar{\tau})]^{2} d \sigma \neq 0
$$

for all $t>0$. They are globally distinguishable if the above inequality is satisfied for all $\tau$ and $\bar{\tau}$ belonging to $\mathbb{R}_{+}$, and locally distinguishable if it is satisfied only for $\tau$ and $\bar{\tau}$ belonging to a set $\left[\tau_{\min }, \tau_{\max }\right]$.

Equivalently, $\tau$ is said to be identifiable if there exists an interval $T>0$ such that

$$
\int_{t-T}^{t}[P(z, \sigma, \tau)-P(z, \sigma, \bar{\tau})]^{2} d \sigma=0 \text { implies } \tau=\bar{\tau}
$$

for all $t>0$. The identifiability is global if (16) is satisfied for all $\bar{\tau} \in \mathbb{R}_{+}$, and it is local identifiable if (16) is satisfied only for $\bar{\tau} \in\left[\tau_{\min }, \tau_{\max }\right]$ where $\left[\tau_{\min }, \tau_{\max }\right]$ is the neighborhood of $\tau$.

Let us remark that Theorem 1 guarantees only that the $\rho$ th equation of (13) contains the delay in an essential way, which is only a necessary condition to identify the delay.

For the concrete signal $P(z, t, \tau)$ of (13) under Assumption 1 and 2 , the delay identifiability can be analyzed for three different types.

- Constant signal: It is easy to see that, in this situation, we cannot find a $T>0$ such that (16) is satisfied for all $t>0$, thus $\tau$ is not identifiable, and this coincides with the wellknown result.

- Periodic signal: Let $T_{p}$ denote as the period of $P(z, t, \tau)$, thus $P(z, t, \tau)=P\left(z, t+T_{P}, \tau\right)$. In this case, it is clear that $\tau$ and $k T_{p}+\tau$ for $k \in \mathbb{N}$ are not globally distinguishable. However, by limiting the delay in a bounded set, then we can have a weak and local definition. Precisely, if there exists an interval $T>0$ such that (16) is satisfied for all $t>$ 0 , and for $\tau \in\left[\tau_{\min }, \tau_{\max }\right]$ and $\bar{\tau} \in\left[\tau_{\min }, \tau_{\max }\right]$ with $\tau_{\max }<$ $T_{p}$, then $\tau$ in (13) can be locally identifiable with $\tau=\bar{\tau}$. 
- Aperiodic signal: Since $P(z, t, \tau)$ is aperiodic, then $T_{p}=$ $+\infty$. The satisfaction of (16) for all $t>0$ directly imposes $\tau=\bar{\tau}$ for any $\tau, \bar{\tau} \in \mathbb{R}_{+}$, thus for any aperiodic signal, its delay can be always globally identifiable.

In the following, the situation of a constant signal will be excluded, and we are going to use a sliding mode technique to identify the delay.

\section{Delay identification via sliding mode}

In order to identify the bounded delay $\tau \in\left[\tau_{\min }, \tau_{\max }\right]$ with $\tau_{\max }<T_{p}$ in (13), it is needed to make the following assumption.

Assumption 3. It is assumed that $\tau$ in (13) is at least locally identifiable, and that for $\bar{\tau} \neq \tau$ with $\bar{\tau} \in\left[\tau_{\min }, \tau_{\max }\right]$, there exists an interval $T>0$ such that

$$
\int_{t-T}^{t} \frac{P(z, \sigma, \tau)-P(z, \sigma, \bar{\tau})}{\tau-\bar{\tau}} \frac{\partial P(z, \sigma, \bar{\tau})}{\partial \bar{\tau}} d \sigma \neq 0
$$

for all $t>0$.

Remark 2. The above inequality is similar to the Persistent Excitation $(P E)$ condition required in parameter identification. It is clear that, when $\tau \rightarrow \bar{\tau}$, (17) is equivalent to

$$
\int_{t-T}^{t}\left[\frac{\partial P(z, \sigma, \bar{\tau})}{\partial \bar{\tau}}\right]^{2} d \sigma \neq 0, \forall t>0
$$

The above inequality implies that the variation of $\bar{\tau}$ in $P(z, \sigma, \bar{\tau})$ cannot be always equal to zero, which excludes clearly the constant signal whose delay is not identifiable.

Remark 3. We can notice as well that, for different $\bar{\tau}$ and $\tau$, inequality (17) implies there exists an interval $T>0$ such that

$$
\int_{t-T}^{t}(P(z, \sigma, \tau)-P(z, \sigma, \bar{\tau})) \frac{\partial P(z, \sigma, \bar{\tau})}{\partial \bar{\tau}} d \sigma \neq 0, \forall t>0
$$

It is worth noting that this condition is less restrictive than those stated in the literature. For example, in Diop et al. (2001) and Drakunov et al. (2006) when treating identification of a single time delay for linear systems, the authors imposed the strictly monotonic condition: $\left|\frac{\partial P(z, t, \tau)}{\partial t}\right|>0, \forall t$. It is clear that those methods cannot be applied to treat periodic signals (not monotonic). The introduction of the integral over an interval is naturally inspired by the PE condition of parameter identification to cover both monotonic and non-monotonic cases. Similar to the PE condition, this integral condition (17) should be non-zero in order to able to identify the delay using different methods. This is the key assumption of the continuous Newton method Saupe (1988) used in this paper.

Considering the bounded delay $\tau \in\left[\tau_{\min }, \tau_{\max }\right]$ in (13), let $\hat{\tau}$ denote as the estimation of $\tau$. Since the transformed canonical form (13) contains $z_{i}=y^{(i)}$ for $1 \leq i \leq \rho$, it is natural to use a differentiator to estimate those $z_{i}$. Based on this thinking, a delay estimator is proposed in (19)) which contains two parts: the first part is a high order sliding mode differentiator (the first $\rho+1$ equations of (19)) Levant (2003) and the second part is a modified continuous Newton method.

$$
\left\{\begin{aligned}
\dot{\hat{z}}_{1} & =\hat{z}_{2}-\lambda_{\rho+1}\left|\hat{z}_{1}-y\right|^{\frac{\rho}{\rho+1}} \operatorname{sign}\left(\hat{z}_{1}-y\right) \\
\dot{z}_{2} & =\hat{z}_{3}-\lambda_{\rho}\left|\hat{z}_{1}-y\right|^{\frac{\rho-1}{\rho+1}} \operatorname{sign}\left(\hat{z}_{1}-y\right) \\
\vdots & \\
\dot{z}_{\rho} & =\hat{z}_{\rho+1}-\lambda_{2}\left|\hat{z}_{1}-y\right|^{\frac{1}{\rho+1}} \operatorname{sign}\left(\hat{z}_{1}-y\right) \\
\dot{z}_{\rho+1} & =-\lambda_{1} \operatorname{sign}\left(\hat{z}_{1}-y\right) \\
\dot{\hat{\tau}} & =\frac{K S_{\hat{z}}(t, \hat{\tau})+E_{T \Delta^{2}} \operatorname{Step}\left(E_{\left.T \Delta^{2}, t\right)}\right.}{2 \int_{t-T}^{t} \Delta_{\hat{z}}(\sigma, \hat{\tau}) \frac{\partial P(\hat{z}, \sigma, \hat{\tau})}{\partial \hat{\tau}} d \sigma}, \hat{\tau}(0)>0
\end{aligned}\right.
$$

where $\hat{\tau}$ is initialized with positive initial functions. $\lambda_{l}$ for $1 \leq$ $l \leq \rho+1$ and $K$ are positive constants. $P(\hat{z}, \sigma, \hat{\tau})$ was given in (15) by replacing $z$ via $\hat{z}$, with

$$
\begin{gathered}
\Delta_{\hat{z}}(\sigma, \hat{\tau})=\hat{z}_{\rho+1}(\sigma)-P(\hat{z}, \sigma, \hat{\tau}) \\
S_{\hat{z}}(t, \hat{\tau})=\int_{t-T}^{t}\left[\Delta_{\hat{z}}(\sigma, \hat{\tau})\right]^{2} d \sigma \\
E_{T \Delta^{2}}=\Delta_{\hat{z}}^{2}(t, \hat{\tau})-\Delta_{\hat{z}}^{2}(t-T, \hat{\tau})
\end{gathered}
$$

and $\operatorname{Step}\left(E_{T \Delta^{2}}, t\right)$ is the standard Heaviside function

Step $(*)= \begin{cases}1, & \text { if } E_{T \Delta^{2}}>0 \text { and } t>\max \left\{T_{s}, T+d_{\max } \hat{\tau}(0)\right\} \\ 0, & \text { otherwise }\end{cases}$

where $T$ is the integral interval defined in (17). $d_{\max }$ is given in (14) and $T_{S}$ represents the settling time of the high order sliding mode differentiator in (19). This function is used to guarantee that $\hat{\tau}$ is always non-negative.

Remark 4. Due to Assumption $1, x(t)$ is bounded on $W \subset \mathbb{R}^{n}$, therefore $z(t)$ is bounded as well. So, for any initial condition $\hat{z}(0)$ taken in (19), there always exists a settling time $T_{s}$ such that for all $t \geq T_{s}$ we have $\hat{z}_{i}(t)=y^{(i)}(t)$ for $1 \leq i \leq \rho+1$. The procedure to tune and choose the parameters $\bar{\lambda}_{i}$ is presented in the appendix. The last equation in (19) is a modified continuous Newton method for the purpose of identifying the delay.

Theorem 2. Supposed Assumptions 1, 2 and 3 are all satisfied for system (6), then there exist positive constants $\lambda_{l}$ for $1 \leq$ $l \leq \rho+1$ and $K>0$ such that $\hat{\tau}$ in (19) locally exponentially converges to $\tau$.

Proof. According to Assumption 1, system (6) admits a unique smooth solution on the compact set $W$, thus $y^{(i)}(t)$ for $1 \leq i \leq \rho$ and $t \in[-s \tau, 0]$ exists and is bounded on $W$. Therefore, there exists a Lipschitz constant $L$ such that $\left\|y^{(\rho+1)}\right\| \leq L$. Assumption 2 guarantees that the delay $\tau$ is involved in the output delay equation in an essential way, thus this can be used to identify the delay. Consider now the transformed normal form (13). According to Levant (2003), the dynamics $\hat{z}$ defined in (19) is a high order sliding mode differentiator. With some properly chosen gains $\lambda_{l}$ for $1 \leq l \leq \rho+1$, it has been proven that this differentiator converges in a finite time $T_{s}$, i.e., for all $t \geq T_{S}$ we have $\hat{z}_{i}(t)=z_{i}(t)$ for $1 \leq i \leq \rho$ and $\hat{z}_{j}=y^{(j-1)}(t)$ for $1 \leq j \leq \rho+1$. 
Therefore, when $t>\max \left\{T_{s}, T+d_{\max } \hat{\tau}(0)\right\}$, replacing $\hat{z}_{i}$ in (20) by $z_{i}$ for $1 \leq i \leq \rho$, we have

$$
P(\hat{z}, t, \hat{\tau})=P(z, t, \hat{\tau})
$$

and

$$
\begin{aligned}
\Delta_{\hat{z}}(t, \hat{\tau}) & =\hat{z}_{\rho+1}(t)-P(\hat{z}, t, \hat{\tau}) \\
& =P(z, t, \tau)-P(z, t, \hat{\tau}) \triangleq \Delta_{z}(t, \hat{\tau})
\end{aligned}
$$

where $\Delta_{z}(t, \hat{\tau})$ was defined as the difference between $P(z, t, \tau)$ and $P(z, t, \hat{\tau})$. Thus,

$$
S_{\hat{z}}(t, \hat{\tau})=\int_{t-T}^{t}\left[\Delta_{z}(\sigma, \hat{\tau})\right]^{2} d \sigma, \forall t>\max \left\{T_{s}, T+d_{\max } \hat{\tau}(0)\right\}
$$

which yields

$$
\frac{\partial S_{\hat{z}}(t, \hat{\tau})}{\partial t}=\Delta_{z}^{2}(t, \hat{\tau})-\Delta_{z}^{2}(t-T, \hat{\tau})
$$

and

$$
\frac{\partial S_{\hat{z}}(t, \hat{\tau})}{\partial \hat{\tau}}=-2 \int_{t-T}^{t} \Delta_{z}(\sigma, \hat{\tau}) \frac{\partial P(z, \sigma, \hat{\tau})}{\partial \hat{\tau}} d \sigma
$$

so we have

$$
\begin{aligned}
\frac{d S_{\hat{z}}(t, \hat{\tau})}{d t}= & \frac{\partial S_{\hat{z}}(t, \hat{\tau})}{\partial t}+\frac{\partial S_{\hat{z}}(t, \hat{\tau})}{\partial \hat{\tau}} \dot{\tau} \\
= & \Delta_{z}^{2}(t, \hat{\tau})-\Delta_{z}^{2}(t-T, \hat{\tau}) \\
& -2 \int_{t-T}^{t} \Delta_{z}(\sigma, \hat{\tau}) \frac{\partial P(z, \sigma, \hat{\tau})}{\partial \hat{\tau}} d \sigma \times \dot{\hat{\tau}} \\
= & E_{T \Delta^{2}}-2 \int_{t-T}^{t} \Delta_{\hat{z}}(\sigma, \hat{\tau}) \frac{\partial P(\hat{z}, \sigma, \hat{\tau})}{\partial \hat{\tau}} d \sigma \times \dot{\tau}
\end{aligned}
$$

Due to the fact that Assumption 3 is satisfied, then $\int_{t-T}^{t} \Delta_{z}(\sigma, \hat{\tau}) \frac{\partial P(z, \sigma, \hat{\tau})}{\partial \hat{\tau}} d \sigma$ is invertible if $\hat{\tau} \neq \tau$. By defining

$$
\dot{\hat{\tau}}=\frac{K S_{\hat{z}}(t, \hat{\tau})+E_{T \Delta^{2}} \operatorname{Step}\left(E_{T \Delta^{2}}, t\right)}{2 \int_{t-T}^{t} \Delta_{\hat{z}}(\sigma, \hat{\tau}) \frac{\partial P(\hat{z}, \sigma, \hat{\tau})}{\partial \hat{\tau}} d \sigma}
$$

we obtain

$$
\begin{aligned}
\frac{d S_{\hat{z}}(t, \hat{\tau})}{d t} & =-K S_{\hat{z}}+E_{T \Delta^{2}}\left[1-\operatorname{Step}\left(E_{T \Delta^{2}}, t\right)\right] \\
& = \begin{cases}-K S_{\hat{z}}, & \text { if } E_{T \Delta^{2}}>0 \\
-K S_{\hat{z}}+E_{T \Delta^{2}}, & \text { if } E_{T \Delta^{2}} \leq 0\end{cases}
\end{aligned}
$$

thus, for both two cases $S_{\hat{z}}(t, \hat{\tau})$ exponentially converges to zero. This implies that

$$
\lim _{t \rightarrow \infty} \int_{t-T}^{t}\left[\Delta_{z}(\sigma, \hat{\tau})\right]^{2} d \sigma=0
$$

and according to Definition 2, we have $\hat{\tau}$ exponentially converging to $\tau$. Notice that when $\hat{\tau} \rightarrow \tau$ where $\tau$ is positive, we get

$$
\begin{aligned}
& \lim _{\hat{\tau} \rightarrow \tau} \frac{K S_{\hat{z}}(t, \hat{\tau})+\Delta_{\hat{\hat{z}}}^{2}(t, \hat{\tau})-\Delta_{\hat{z}}^{2}(t-T, \hat{\tau})}{2 \int_{t-T}^{t} \Delta_{\hat{z}}(\sigma, \hat{\tau}) \frac{\partial P(\hat{z}, \sigma, \hat{\tau})}{\partial \hat{\tau}} d \sigma} \\
& =\lim _{\hat{\tau} \rightarrow \tau}(\tau-\hat{\tau}) \frac{K \frac{S_{\hat{\mathcal{Z}}}(t, \hat{\tau})}{(\tau-\hat{\tau})^{2}}+\frac{\Delta_{\hat{z}}^{2}(t, \hat{\tau})-\Delta_{\hat{Z}}^{2}(t-T, \hat{\tau})}{(\tau-\hat{\tau})^{2}}}{2 \int_{t-T}^{t} \frac{\Delta_{\hat{Z}}(\sigma, \hat{\tau})}{\tau-\hat{\tau}} \frac{\partial P(\hat{z}, \sigma, \hat{\tau})}{\partial \hat{\tau}} d \sigma}
\end{aligned}
$$

Due to Assumption 3, we have the following relations:

$$
\lim _{\hat{\tau} \rightarrow \tau} \int_{t-T}^{t} \frac{\Delta_{\hat{z}}(\sigma, \hat{\tau})}{\tau-\hat{\tau}} \frac{\partial P(\hat{z}, \sigma, \hat{\tau})}{\partial \hat{\tau}} d \sigma=\int_{t-T}^{t}\left[\frac{\partial P(\hat{z}, \sigma, \hat{\tau})}{\partial \hat{\tau}}\right]^{2} d \sigma>0
$$

$$
\begin{aligned}
\lim _{\hat{\tau} \rightarrow \tau} \frac{S_{\hat{z}}(t, \hat{\tau})}{(\tau-\hat{\tau})^{2}}= & \lim _{\hat{\tau} \rightarrow \tau} \int_{t-T}^{t}\left[\frac{\Delta_{\hat{z}}(\sigma, \hat{\tau})}{(\tau-\hat{\tau})}\right]^{2} d \sigma \\
& =\int_{t-T}^{t}\left[\frac{\partial P(\hat{z}, \sigma, \hat{\tau})}{\partial \hat{\tau}}\right]^{2} d \sigma>0
\end{aligned}
$$

and

$$
\lim _{\hat{\tau} \rightarrow \tau} \frac{\Delta_{\hat{z}}^{2}(t, \hat{\tau})-\Delta_{\hat{\imath}}^{2}(t-T, \hat{\tau})}{(\tau-\hat{\tau})^{2}}=\left[\frac{\partial P(\hat{z}, t, \hat{\tau})}{\partial \hat{\tau}}\right]^{2}-\left[\frac{\partial P(\hat{z}, t-T, \hat{\tau})}{\partial \hat{\tau}}\right]^{2}
$$

which is bounded, since according to Assumption 1 the system (1) admits a smooth and bounded solution. Finally we conclude that

$$
\lim _{\hat{\tau} \rightarrow \tau} \frac{K S_{\hat{\imath}}(t, \hat{\tau})+\Delta_{\hat{\mathcal{L}}}^{2}(t, \hat{\tau})-\Delta_{\hat{\hat{z}}}^{2}(t-T, \hat{\tau})}{2 \int_{t-T}^{t} \Delta_{\hat{z}}(\sigma, \hat{\tau}) \frac{\partial P(\hat{\bar{z}}, \sigma, \hat{\tau})}{\partial \hat{\tau}} d \sigma}=0
$$

which implies that $\lim _{t \rightarrow \infty} \dot{\hat{\tau}}(t)=0$ and $\lim _{t \rightarrow \infty} \hat{\tau}(t)=\tau$.

Remark 5. Compared to other existing methods, such as Diop et al. (2001) and Drakunov et al. (2006) which can only treat the monotonic case, the main novelty of the proposed method is that we can treat periodic signals. This is due to the introduction of the integral condition (17). Moreover, since sliding mode technique has been used which is well known for its robustness with respect to disturbance, the proposed delay estimator benefits as well such property of robustness compared to other non-variable structure methods.

Remark 6. It is worth noting that finite-time estimation of $\tau$ can be achieved by simply modifying the dynamics $\dot{\hat{\tau}}$ in (19) as follows:

$$
\dot{\hat{\tau}}=\frac{K \sqrt{S_{\hat{z}}(t, \hat{\tau})}+E_{T \Delta^{2}} \operatorname{Step}\left(E_{T \Delta^{2}}, t\right)}{2 \int_{t-T}^{t} \Delta_{\hat{z}}(\sigma, \hat{\tau}) \frac{\partial P(\hat{z}, \sigma, \hat{\tau})}{\partial \hat{\tau}} d \sigma}
$$

By using the above dynamics and following the same proof idea as for Theorem 2, we can obtain

$$
\frac{d S_{\hat{z}}(t, \hat{\tau})}{d t}=-K \sqrt{S_{\hat{z}}(t, \hat{\tau})}+E_{T \Delta^{2}}\left[1-\operatorname{Step}\left(E_{T \Delta^{2}}, t\right)\right]
$$

and thus $S_{\hat{z}}(t, \hat{\tau})$ converges to zero in a finite time.

\section{Illustrative example}

Consider the following academic example:

$$
\left\{\begin{aligned}
\dot{x}_{1}(t)= & -x_{1}^{2}(t-2 \tau)+x_{2}(t) \\
\dot{x}_{2}(t)= & -x_{1}(t)-2 x_{1}(t-2 \tau) x_{1}^{2}(t-4 \tau) \\
& +2 x_{1}^{2}(t-2 \tau) x_{2}^{2}(t-2 \tau) \\
& -\sin \left(-x_{1}(t-2 \tau) x_{1}(t-3 \tau)\right) \\
\dot{x}_{3}(t)= & -x_{3}(t)+\sin x_{1}(t-3 \tau) \\
y(t)= & x_{1}(t-\tau)
\end{aligned}\right.
$$

Introducing the delay operator $\delta$, the above example can be written as the compact form of (6) as follows:

$$
\left\{\begin{array}{l}
\dot{x}_{1}=-\delta^{2} x_{1}^{2}+x_{2} \\
\dot{x}_{2}=-x_{1}-2 \delta^{2} x_{1} \delta^{4} x_{1}^{2}+2 \delta^{2} x_{1} \delta^{2} x_{2}-\sin \left(\delta^{2} x_{1} \delta^{3} x_{1}\right) \\
\dot{x}_{3}=-x_{3}+\delta^{3} \sin x_{1} \\
y=\delta x_{1}
\end{array}\right.
$$


It can be easily checked that the observability index for $y$ is $\rho=2$, thus, according to (10), $\ddot{y}=L_{f}^{\rho} h=L_{f}^{2} h$ is an output delay equation. In order to check inequality (11), we calculate

$$
\operatorname{rank}_{\mathscr{K}(\delta]} \frac{\partial\left\{h, L_{f} h\right\}}{\partial x}=2
$$

and

$$
\operatorname{rank}_{\mathscr{K}} \frac{\partial\left\{h, L_{f} h, L_{f}^{\rho} h\right\}}{\partial x}=3
$$

and according to Theorem 1, the delay involved in (25) is identifiable.

Following (12), we note $\Phi_{h}=\left[\begin{array}{c}h \\ L_{f} h\end{array}\right]=\left[\begin{array}{c}\delta x_{1} \\ -\delta^{3} x_{1}^{2}+\delta x_{2}\end{array}\right]$ and select $\Phi_{\xi}=x_{3}$. Then the change of coordinates $\Phi=$ $\left[\begin{array}{c}\Phi_{h} \\ \Phi_{\xi}\end{array}\right]$ brings the studied system into:

$$
\left\{\begin{array}{l}
\dot{z}_{1}=z_{2} \\
\dot{z}_{2}=-z_{1}-\sin \left(\delta z_{1} \delta^{2} z_{1}\right) \\
\dot{\xi}=-\xi+\delta^{2} \sin z_{1} \\
y=z_{1}
\end{array}\right.
$$

According to (15), the output delay equation for the example can be written as

$$
\dot{z}_{2}(t)=P(z, t, \tau)
$$

with $P(z, t, \tau)=-z_{1}(t)-\sin \left(z_{1}(t-\tau) z_{1}(t-2 \tau)\right)$ for which we can apply the proposed delay estimator described in (19). For the simulation, we can implement the proposed estimator as follows:

$$
\left\{\begin{aligned}
\dot{z}_{1} & =\hat{z}_{2}-\lambda_{3}\left|\hat{z}_{1}-y\right|^{\frac{2}{3}} \operatorname{sign}\left(\hat{z}_{1}-y\right) \\
\dot{z}_{2} & =\hat{z}_{3}-\lambda_{2}\left|\hat{z}_{1}-y\right|^{\frac{1}{3}} \operatorname{sign}\left(\hat{z}_{1}-y\right) \\
\dot{\hat{z}}_{3} & =-\lambda_{1} \operatorname{sign}\left(\hat{z}_{1}-y\right) \\
\dot{\hat{\tau}} & =\frac{K S_{\hat{z}}(t, \hat{\tau})+E_{T \Delta^{2}} \operatorname{Step}\left(E_{\left.T \Delta^{2}, t\right)}\right.}{2 \int_{t-T}^{t} \Delta_{\hat{z}}(\sigma, \hat{\tau}) \frac{\partial P(\hat{z}, \sigma, \hat{\tau})}{\partial \hat{\tau}} d \sigma}
\end{aligned}\right.
$$

with

$$
\begin{gathered}
\Delta_{\hat{z}}(\sigma, \hat{\tau})=\hat{z}_{3}(\sigma)+\hat{z}_{1}(\sigma)+\sin \left(\hat{z}_{1}(\sigma-\hat{\tau}) \hat{z}_{1}(\sigma-2 \hat{\tau})\right) \\
E_{T \Delta^{2}}=\Delta_{\hat{z}}^{2}(t, \hat{\tau})-\Delta_{\hat{z}}^{2}(t-T, \hat{\tau}) \\
\frac{\partial P(\hat{z}, \sigma, \hat{\tau})}{\partial \hat{\tau}}=\begin{array}{l}
\hat{z}_{2}(\sigma-\hat{\tau}) \hat{z}_{1}(\sigma-2 \hat{\tau}) \cos \left(\hat{z}_{1}(\sigma-\hat{\tau}) \hat{z}_{1}(\sigma-2 \hat{\tau})\right) \\
+2 \hat{z}_{1}(\sigma-\hat{\tau}) \hat{z}_{2}(\sigma-2 \hat{\tau}) \cos \left(\hat{z}_{1}(\sigma-\hat{\tau}) \hat{z}_{1}(\sigma-2 \hat{\tau})\right)
\end{array}
\end{gathered}
$$

and

$$
S_{\hat{z}}(t, \hat{\tau})=\int_{t-T}^{t}\left[\Delta_{\hat{z}}(\sigma, \hat{\tau})\right]^{2} d \sigma
$$

According to results presented in the appendix, the parameters of the estimator have been selected as an appropriate solution of a linear matrix inequality (28), which yields $\lambda=[52.9566,3423.7803,52248.0756]$ for $\alpha=1, \eta=(1+$ $\sqrt{5}) /(3+\sqrt{5})$ and $L=30$. The corresponding simulation results are given with $K=2, \tau=0.5 s$, and $T=2 s$. Fig. 1 and Fig. 2 illustrate that the proposed estimator can estimate $z_{1}$ and $z_{2}$ in a finite time for the case where the initial condition of $\hat{\tau}$ is equal to $0.4 s$. Fig. 3 shows that Assumption 3 is satisfied, and

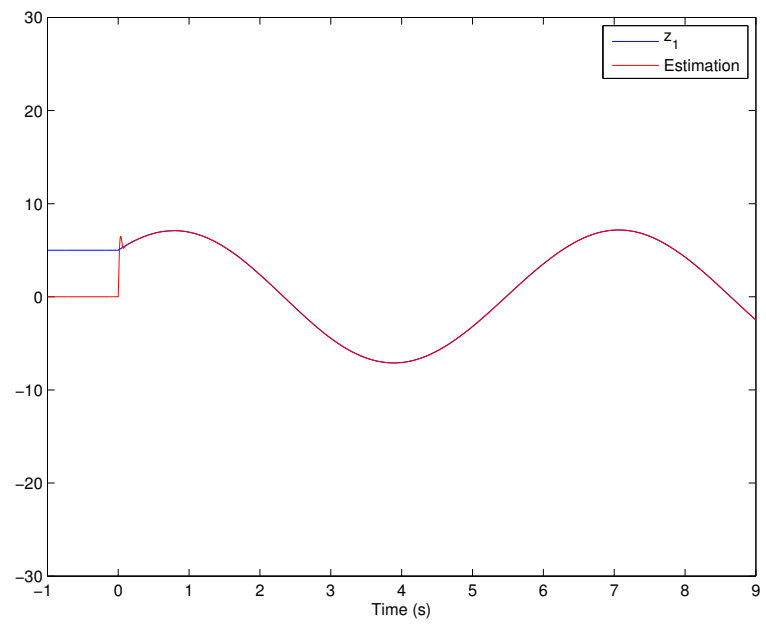

Figure 1: The trajectory $z_{1}$ of (27) and its estimation $\hat{z}_{1}$ when $\tau=0.5 s$

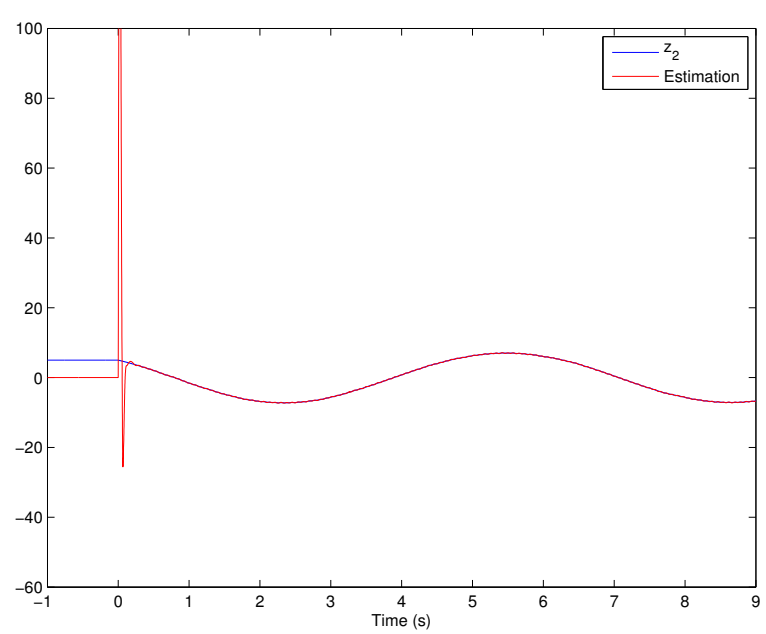

Figure 2: The trajectory $z_{2}$ of (27) and its estimation $\hat{z}_{2}$ when $\tau=0.5 s$

Fig. 4 depicts the delay estimation. The same results are given in Fig. 5 and 6 for the case where the initial condition of $\hat{\tau}$ is equal to $0.6 s$. According to the definition of the Heaviside function in (19), the large initial condition of $\hat{\tau}(0)$ will yield a late activation of the estimator, and this phenomenon can be noticed as well for those two simulations, where the estimation process for $\hat{\tau}(0)=0.6 s$ starts a little later than that for $\hat{\tau}(0)=0.4 s$.

\section{Conclusion}

Delay identification is not an easy task even for a linear time-delay system. This paper studied this issue for a class of nonlinear time-delay systems. We have firstly used the noncommutative rings to deduce an output delay equation, and then given necessary and sufficient condition to judge whether this equation contains the delay in an essential way. After that, the definition of delay identifiability was given and has been 


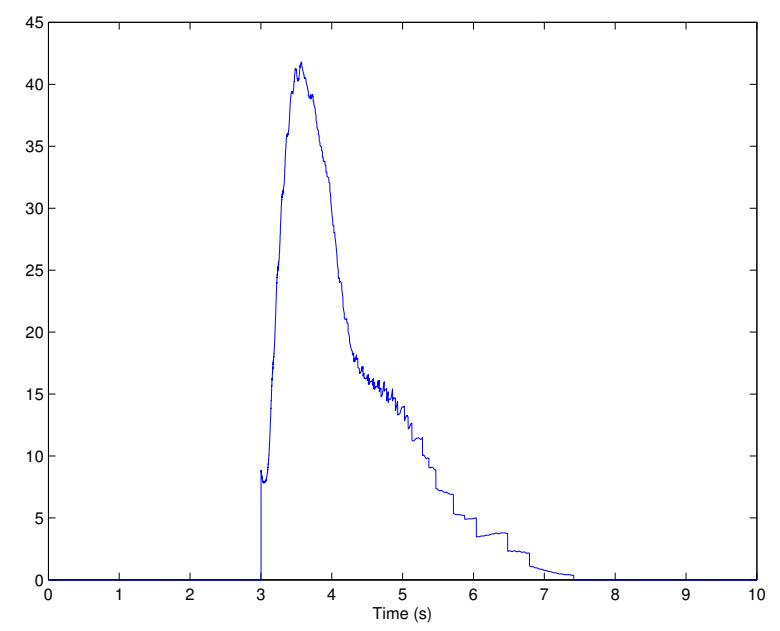

Figure 3: Verification of Assumption 3 when $\hat{\tau}$ is initialized as $0.4 s$

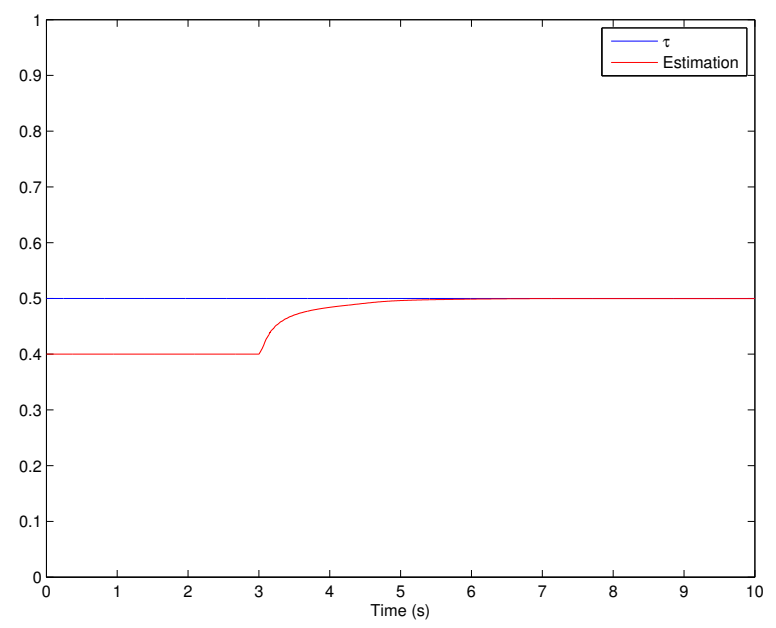

Figure 4: $\tau=0.5 s$ and its estimation $\hat{\tau}$ with initial condition equal to $0.4 s$

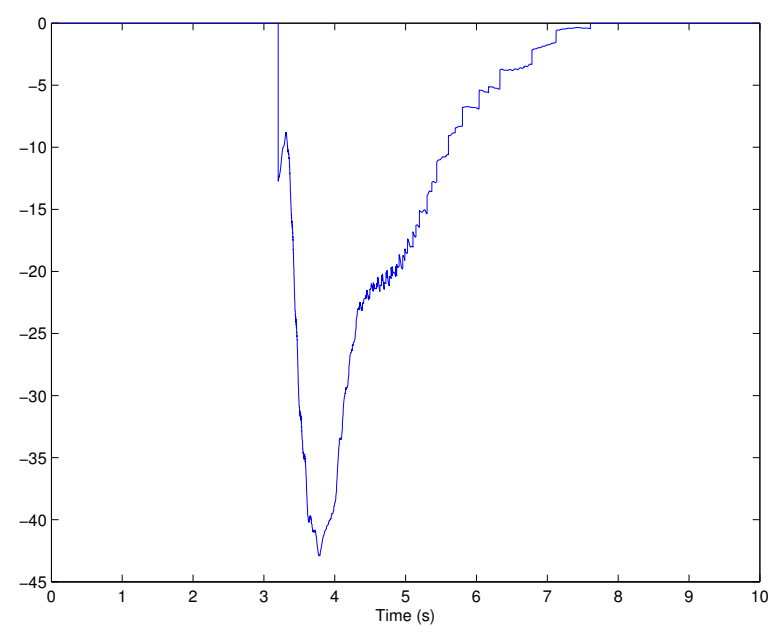

Figure 5: Verification of Assumption 3 when $\hat{\tau}$ is initialized as $0.6 s$

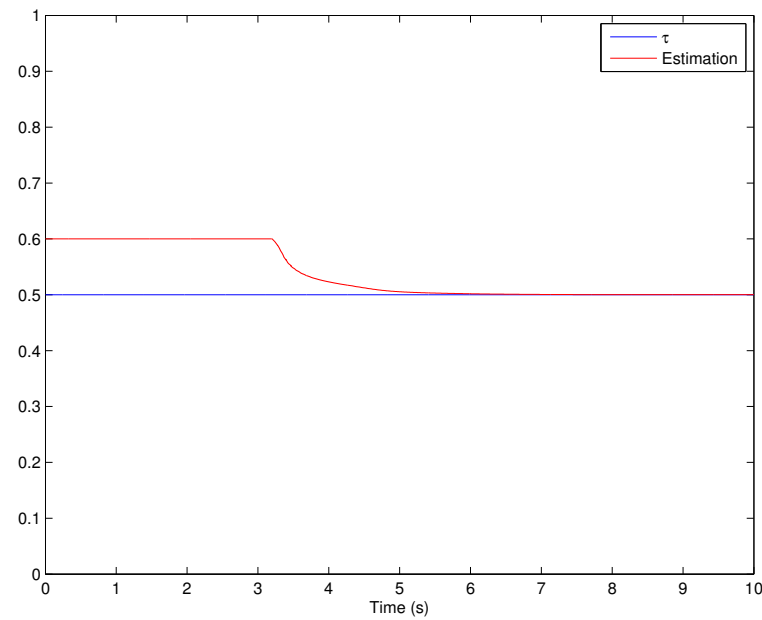

Figure 6: $\tau=0.5 s$ and its estimation $\hat{\tau}$ with initial condition equal to $0.6 s$

analyzed for three different situations (constant, periodic and aperiodic). Concerning the estimation method, we combined the high order sliding mode technique and the classical Newton method. The sliding mode technique enables us to have a finite-time estimation of the successive derivatives of the measurements. After the finite time convergence, it permits us to define an objective for which the classical Newton method was applied to estimate the delay.

\section{Appendix}

The problem of the tuning of the gains of the high order sliding mode differentiator has been studied in Polyakov et al. (2014) using the so-called Implicit Lyapunov Function Method Polyakov et al. (2015).

Let us consider the error equation for the finite-time differentiator defined by the first $\rho+1$ equation of (19), which can be rewritten as follows:

$$
\dot{e}=\left(A+\tilde{D}\left(\left|e_{1}\right|^{-1}\right) \lambda C\right) e+B y^{(\rho+1)},
$$

where

$$
\begin{aligned}
& e=\left(z_{1}-y, z_{2}-\dot{y}, \cdots, z_{\rho+1}-y^{(\rho)}\right)^{T} \\
& B=(0, \cdots, 0,1)^{T} \\
& C=(1,0, \cdots, 0) \\
& \lambda=\left(-\lambda_{1}, \cdots,-\lambda_{\rho+1}\right)^{T}
\end{aligned}
$$

and

$$
\begin{gathered}
A=\left(\begin{array}{cccccc}
0 & 1 & 0 & 0 & \cdots & 0 \\
0 & 0 & 1 & 0 & \cdots & 0 \\
\vdots & \vdots & \vdots & \vdots & \ddots & \vdots \\
0 & 0 & 0 & 0 & \cdots & \vdots \\
0 & 0 & 0 & 0 & \cdots & 0
\end{array}\right) \\
\tilde{D}(\theta)=\operatorname{diag}\left(\theta^{\frac{1}{\rho+1}}, \theta^{\frac{2}{\rho+1}}, \cdots, \theta\right)
\end{gathered}
$$

with $\theta>0$.

A Lyapunov function candidate for the obtained system can be defined in the implicit form $Q(V, e)=0$, where

$$
Q(V, e)=e^{T} D\left(V^{-1}\right) P D\left(V^{-1}\right) e-1
$$


and

$$
D(\theta)=\operatorname{diag}\left(\theta^{r_{1}}, \theta^{r_{2}}, \cdots, \theta^{r_{\rho+1}}\right)
$$

with $\theta>0$ and $r_{j}=1-(j-1) /(\rho+1)$.

Let us denote

$$
\begin{aligned}
& E=\operatorname{diag}\{1,0, \cdots, 0\} \\
& H=\operatorname{diag}\left(r_{1}, \cdots, r_{\rho+1}\right)
\end{aligned}
$$

and

$$
\Xi(\theta)=\theta \tilde{D}\left(\theta^{-1}\right)-\theta I
$$

with $\theta>0$ and assume that $\left|y^{(\rho+1)}\right| \leq L$. If the system of linear matrix inequalities

$$
\begin{aligned}
& \begin{array}{c}
W:=\left(\begin{array}{ccc}
P A+A^{T} P+Y C+C^{T} Y^{T}+(1+L) P+\alpha(H P+P H) & P & P B \\
P & -Z & 0 \\
B^{T} P & 0 & -L^{-1}
\end{array}\right) \leq 0, \\
\left(\begin{array}{cc}
1 & Y_{P}^{T} \\
Y & P
\end{array}\right) \geq 0, \quad H P+P H>0, \quad P \geq \eta E_{1} P E_{1}, \quad P>0, \quad Z>0,
\end{array} \\
& \Theta(\theta):=\Xi(\theta) Z \Xi(\theta) \leq P, \quad \forall \theta \in\left[0, \eta^{-0.5}\right],
\end{aligned}
$$

is feasible for some $\eta \in(0,1), \alpha>0, P \in \mathbb{R}^{(\rho+1) \times(\rho+1)}$, $Y:=P \lambda \in \mathbb{R}^{\rho+1}$ then a function $V: \mathbb{R}^{\rho+1} \rightarrow \mathbb{R}$ defined by the equation $Q(V, e)=0$ is positive definite and continuously differentiable outside the origin and $\dot{V}=\frac{\partial V}{\partial e} \dot{e}=$ $-\left[\frac{\partial Q}{\partial V}\right]^{-1} \frac{\partial Q}{\partial e} \dot{e} \leq-\alpha V^{1 /(\rho+1)}$. This implies that $e_{i}(t)=0$ for $t \geq(\rho+1) V_{0}^{1 /(\rho+1)} / \alpha$, where $V_{0}>0: Q\left(V_{0}, e(0)\right)=0$.

In order to find a vector of observer parameters $\lambda=P^{-1} Y$ the linear matrix inequality (28) must be solved together with the parametric matrix inequality $\Theta(\theta) \leq P$ for $\theta \in\left[0, \eta^{-0.5}\right]$.

\section{References}

Anguelova, M., Wennberg, B., 2008. State elimination and identifiability of the delay parameter for nonlinear time-delay systems. Automatica 44 (5), 1373-1378.

Barbot, J.-P., Zheng, G., Floquet, T., Boutat, D., Richard, J.-P., 2012. Delay estimation algorithm for nonlinear time-delay systems with unknown inputs. in Proc. of IFAC Workshop on Time Delay Systems.

Belkoura, L., 2005. Identifiability of systems described by convolution equations. Automatica 41 (3), 505-512.

Belkoura, L., Richard, J.-P., Fliess, M., 2009. Parameters estimation of systems with delayed and structured entries. Automatica 45 (5), 1117-1125.

Bjorklund, S., Ljung, L., 2003. A review of time-delay estimation techniques. In: Decision and Control, 2003. Proceedings. 42nd IEEE Conference on. Vol. 3. pp. 2502-2507 Vol.3.

Conte, G., Moog, C., Perdon, A., 1999. Nonlinear control systems: An algebraic setting. Lecture Notes in Control and Information Sciences 242, Springer-Verlag,London.

Diop, S., Kolmanovsky, I., Moraal, P., van Nieuwstadt, M., 2001. Preserving stability/performance when facing an unknown time-delay. Control Engineering Practice 9 (12), 1319 - 1325

Drakunov, S., Perruquetti, W., Richard, J.-P., Belkoura, L., 2006. Delay identification in time-delay systems using variable structure observers. Annual Reviews in Control 30 (2), 143-158.

Ježek, J., 1996. Rings of skew polynomials in algebraical approach to control theory. Kybernetika 32 (1), 63-80.

Kolmanovskii, V., Myshkis, A., 1999. Introduction to the theory and application of functional differential equations. Kluwer Academic Publishers, Dordrecht.

Krener, A., 1985. $\left(\mathrm{A} d_{f, g}\right),\left(a d_{f, g}\right)$ and locally $\left(a d_{f, g}\right)$ invariant and controllability distributions. SIAM Journal on Control and Optimization 23 (4), 523-549.

Levant, A., 2003. Higher-order sliding modes, differentiation and outputfeedback control. International Journal of Control 76 (9-10), 924-941.
Moog, C., Castro-Linares, R., Velasco-Villa, M., Marque-Martinez, L. A., 2000. The disturbance decoupling problem for time-delay nonlinear systems. IEEE Transactions on Automatic Control 45 (2).

Niculescu, S.-I., 2001. Delay effects on stability: A robust control approach. Lecture Notes in Control and Information Sciences, Springer 269.

Orlov, Y., Belkoura, L., Richard, J., Dambrine, M., 2003. Adaptive identification of linear time-delay systems. International Journal of Robust and Nonlinear Control 13 (9), 857-872.

Polyakov, A., Efimov, D., Perruquetti, W., 2014. Homogeneous differentiator design using implicit lyapunov function method. In: European Control Conference.

Polyakov, A., Efimov, D., Perruquetti, W., 2015. Finite-time and fixed-time stabilization: Implicit Lyapunov function approach. Automatica 51, 332 340.

Ren, X., Rad, A., Chan, P., Lo, W., 2005. Online identification of continuoustime systems with unknown time delay. IEEE Transaction on Automatic Control 50 (9), 1418-1422.

Saupe, D., 1988. Discrete versus continuous newton's method: A case study. Acta Applicandae Mathematica 13 (1), 59-80.

Tuch, J., Feuer, A., Palmor, Z., 1994. Time delay estimation in continuous linear time-invariant systems. Automatic Control, IEEE Transactions on 39 (4), $823-827$.

Xia, X., Marquez, L., Zagalak, P., Moog, C., 2002. Analysis of nonlinear time-delay systems using modules over non-commutative rings. Automatica 38 (9), 1549-1555.

Zhang, J., Xia, X., Moog, C., 2006. Parameter identifiability of nonlinear systems with time-delay. IEEE Transactions on Automatic Control 47, 371375 .

Zheng, G., Barbot, J.-P., Boutat, D., 2013. Identification of the delay parameter for nonlinear time-delay systems with unknown inputs. Automatica 49 (6), 1755-1760.

Zheng, G., Barbot, J.-P., Floquet, T., Boutat, D., Richard, J.-P., 2011. On obserability of nonlinear time-delay systems with unknown inputs. IEEE Transactions on Automatic Control 56 (8), 1973-1978.

Zheng, G., Polyakov, A., Levant, A., 2016. Delay estimation for nonlinear timedelay systems. In: Proceedings of the 14th IEEE International Workshop on Variable Structure Systems. pp. 126-130.

Zheng, G., Richard, J.-P., 2016. Identifiability and observability of nonlinear time-delay systems with unknown inputs. In: Karafyllis, I., Malisoff, M., Mazenc, F., Pepe, P. (Eds.), Recent Results on Nonlinear Delay Control Systems. Vol. 4 of Advances in Delays and Dynamics. Springer International Publishing, pp. 385-403. 